\title{
REfine: A Gamified Platform for Participatory Requirements Engineering
}

\author{
Remco Snijders, Fabiano Dalpiaz, \\ and Sjaak Brinkkemper \\ Utrecht University, The Netherlands
}

\author{
Mahmood Hosseini, Raian Ali \\ Bournemouth University, \\ United Kingdom
}

\author{
Atilla Özüm \\ KPMG Technology Advisory, \\ The Netherlands
}

\begin{abstract}
The active involvement of stakeholders in Requirements Engineering (RE) is crucial, for the system under design should fulfill their expectations. In software products, which evolve under the control of Software Product Organizations (SPOs), stakeholders' involvement is limited to SPO representatives and key clients. Thus, key stakeholders are excluded, including current and prospective users. Two emerging trends can help to shift towards a more participatory RE: crowdsourcing eases the access to a large number of stakeholders, and gamification provides means to keep them motivated through feedback loops (that reward the useful participants). In this paper, we build on this potential and propose REfine, a gamified online platform for requirements elicitation and refinement by involving a crowd of stakeholders: users, developers, analysts, etc. We report encouraging results from a case study that show how REfine has led to useful requirements, stakeholders' engagement, and valuable interactions.
\end{abstract}

\section{INTRODUCTION}

The benefits of involving the stakeholders in RE, including customers and users, have been widely acknowledged. Already in 1975, in management science, Zand and Sorensen showed that user participation can overcome resistance to change [1]. Similar results apply to software requirements engineering: user involvement in RE can lead to improved acceptance of a system [2], higher chances of project success [3], greater system understanding by the users [4], improved customer loyalty and broadened market [5].

The contemporary relevance of user involvement is also highlighted by the Standish CHAOS Report [6] on software success and failure, which identifies user involvement as the most important factor for both success and failure in information technology projects. This motivates our research into finding effective ways of engaging users in the RE process.

We consider the case of software products, which are created and evolved under the span of control of software product organizations (SPOs). In this setting, the necessary inputs from users and other stakeholders have to be carefully balanced with the SPO's vision of and roadmap for the product, as well as the business concern of focusing on the key clients that generate most of the revenues.

A possible solution to achieve high, inclusive involvement is crowdsourcing, where a company outsources a function generally performed internally to an undefined (generally large) network of people by means of an open call [7]. Some key activities of the RE process might be outsourced to the stakeholders of the software product. Early attempts in RE already exist. For example, the StakeRare elicitation method [8] makes use of the StakeSource 2.0 tool [9] to involve the crowd in requirements identification and prioritization. A similar approach is taken by the CrowdREquire [10] platform. However, these works do not furnish explicit means to motivate the stakeholders to join and remain in the crowd.

A recent trend that can help improve motivation (ultimately, quality [11]) is gamification: "the use of game design elements in non-game contexts" [12]. Early attempts show that gamification may have a potential in RE: iThink is a game-based collaborative tool [13] that aids in collecting new requirements and gaining feedback on existing requirements.

We make a step further in the direction of gamified and participatory RE by proposing the REfine online platform for eliciting and refining requirements in the context of SPOs. REfine is an essential component of our vision concerning Crowd-Centric Requirements Engineering (CCRE) [14]. In this paper, we make the following contributions:

- We introduce the REfine platform, describing its features and architecture;

- We explain the role of REfine as an element of a method for crowd-centric requirements engineering;

- We report on an initial evaluation of REfine through a case study, where we applied it in the context of a governance risk and compliance tool.

The rest of the paper is structured as follows. In Sec. II, we discuss related work concerning promoting stakeholders' and users' involvement. In Sec. III, we present the REfine tool. In Sec. IV, we explain how REfine fits within CCRE, while we report on its preliminary evaluation in Sec. V. We conclude with a discussion and future directions in Sec. VI.

\section{RELATED WORK}

We review related work about user involvement (Sec. II-A), crowdsourcing (Sec. II-B), and gamification (Sec. II-C).

\section{A. User Involvement}

User involvement has a high impact on software quality. Integrating product development requests from customers improves customer loyalty and might broaden the market [5]. Early user involvement leads to more accurate user requirements, avoiding expensive and unnecessary features and improved system acceptance [2]. User involvement also 
contributes to requirements quality by increasing the chance of project success and lowering the costs of RE [3].

While customer organizations are typically seen as essential, the role of end-users is often underestimated [3], although they are those who will ultimately experience the final quality of the system. However, interacting with users is challenging, especially in terms of gaining access and obtaining consensus [15]. Virtual communities are an increasingly popular solution to carry out different tasks ranging from idea generation to advanced user support (e.g., Get Satisfaction ${ }^{1}$ and UserVoice ${ }^{2}$ ).

\section{B. Crowdsourcing}

An innovative enabler for complex, large-scale user involvement is crowdsourcing, i.e., delegating tasks to a large, undefined network of people through an open call for contribution [7], and then aggregating the results. Brabham [16] observes that a key feature of crowsourcing is the high quality of the wisdom of crowds, in which aggregated average answers could lead to excellent decision making [17].

Specific challenges affect crowdsourcing in terms of quality and motivation. Crowdsourcing responses might be plentiful, but are often noisier than expert data [18]. By focusing on effective task design [19], the quality of the output is enhanced. The motivators of the crowd have also been investigated: studies focusing on iStockphoto [16] and Amazon Mechanical Turk (AMT) [20] identify the opportunity to make money, task autonomy, the development of creative skills and the love and fun of a community as the most important motivators.

In RE, the StakeSource 2.0 tool uses crowdsourcing to identify and prioritize stakeholders and their requirements [9]. The tool also recommends requirements and identifies conflicts, and is supported by the StakeRare method [8]. CrowdREquire [10] and Requirements Bazaar [21] are other solutions along the lines of StakeSource 2.0. These approaches, however, do not focus on the provision of (long-term) incentives. Hosseini et al. [22], [23] identify the quality attributes that affect the effectiveness of crowdsourcing in requirements elicitation, such as largeness, diversity, competence, motivation, etc.

\section{Gamification}

Deterding et al. [12] define gamification as "the use of game design elements in non-game contexts". These elements should be characteristic to games and have a significant role in gameplay. The variety of applications is wide, from a mobile running app where the player is chased by zombies, to expert badges on the Q\&A website Stack Overflow.

Recent research proposes to use gamification within crowdsourcing systems [24]. This idea has precursors in the industry: Threadless lets users score t-shirt models in order to vote them in or out of the shop. By submitting a design, users can win royalties or a gift card for the shop.

Gamification addresses both the motivation and quality challenges of crowdsourcing. For example, in a task where participants perform relevance assessments, with points being

\footnotetext{
${ }^{1}$ https://getsatisfaction.com/

${ }^{2}$ https://www.uservoice.com/
}

achieved when other players agree and when a set of tasks is completed, the gamified approach has led to quicker and higher quality responses with less "cheaters" (users giving useless responses) than non-gamified crowdsourcing [11].

Limited literature exists regarding gamification for RE. Fernandes et al. [13] apply gamification to requirements elicitation by developing the game-based collaborative tool iThink. Preliminary case studies indicate enhanced user involvement in requirements elicitation [25]. While the used surveys were not very rigorous, the results show satisfaction of participants and project managers.

Based on these findings, we argue that gamification can positively impact crowdsourcing and RE, leading to engagement, user satisfaction, innovation, quick and high quality response. However, the game elements that define gamification have to be chosen with great care to obtain this impact.

\section{REfine: Supporting GAMified, CROWD-CENTRIC RE}

REfine is our proposed interactive platform for gamified, crowd-centric requirements engineering ${ }^{3}$. The name refers to Requirements Engineering and the main process that is supported by the platform: refining stakeholders' needs. $R E$ fine allows users to suggest needs, comment on needs and other comments, branch needs and vote for needs and comments. The platform has been built using JavaScript (including jQuery), HTML, CSS, PHP and MySQL. The interface is built on the Bootstrap framework. For the game elements, the Application Programming Interface of PlayBasis was used.

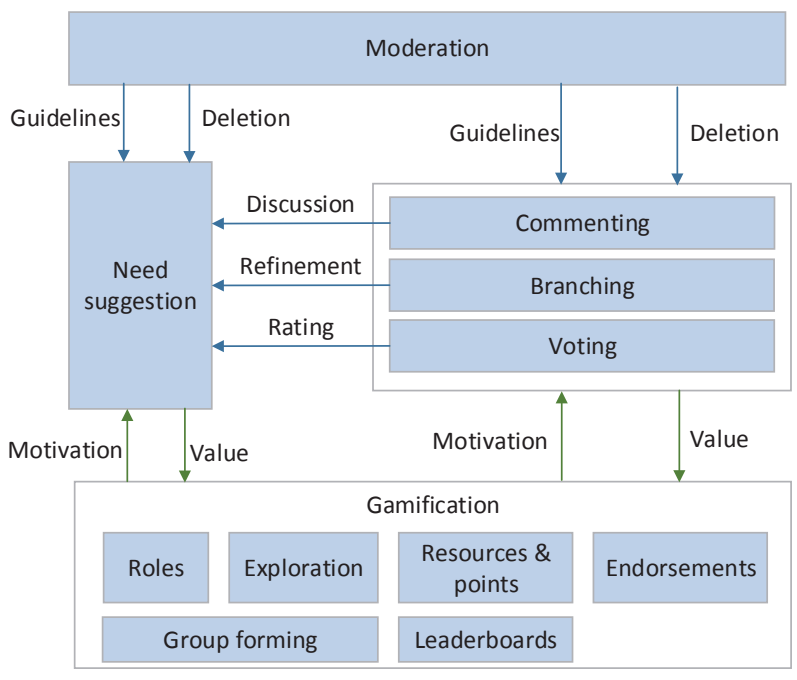

Fig. 1. Functional architecture of REfine

Fig. 1 presents the functional architecture of REfine. A key building block is Need suggestion, which relates to Gamification by creating a feedback loop where the value for the community results in further motivation for the participant. Need suggestion is influenced by Moderation-these functions are used by the community manager-, which focuses on the provision of guidelines and the deletion of irrelevant needs.

\footnotetext{
${ }^{3}$ http://goo.gl/vGxQ47
} 
The other functions that make REfine work are Commenting, Branching and Voting, resulting in further discussion of the needs, their refinement and rating. These functions are also tightly linked with gamification, and subject to moderation.

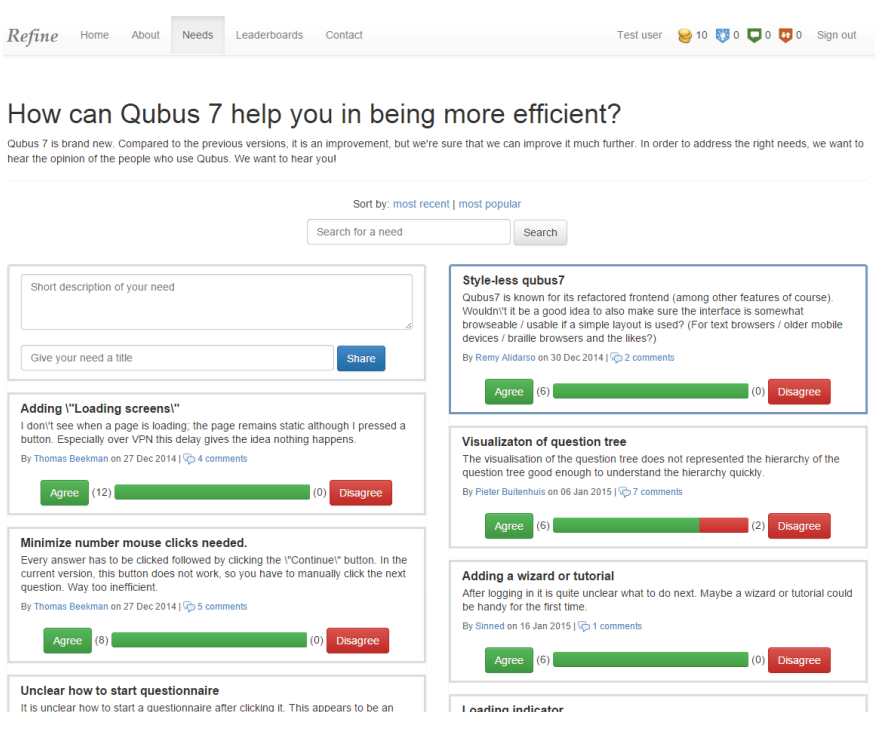

Fig. 2. The needs overview of REfine

Apart from home-, about-, leaderboards- and contact pages, REfine contains three important pages: the needs overview, need details, and user profile. On top of each page, the menu bar can be found. Besides hyperlinks to other pages, the user status bar shows the coins and points of the user. Fig. 2 shows a screenshot of the needs overview.

Six types of game elements were implemented in REfine, which can be related to the social factors that positively influence the attitude towards gamified services [26]. Table I shows the relation between game elements and social factors. We describe each factor in the following.

TABLE I

MAPPING OF GAME ELEMENTS TO RELEVANT SOCIAL FACTORS

\begin{tabular}{|l|c|c|c|c|}
\hline Social Factors & $\begin{array}{l}\text { Network } \\
\text { exposure }\end{array}$ & $\begin{array}{l}\text { Social } \\
\text { influence }\end{array}$ & $\begin{array}{l}\text { Recog- } \\
\text { nition }\end{array}$ & $\begin{array}{l}\text { Reciprocal } \\
\text { benefit }\end{array}$ \\
\hline Roles & $\checkmark$ & & & $\checkmark$ \\
\hline Resources \& points & & & $\checkmark$ & $\checkmark$ \\
\hline Leaderboards & & & $\checkmark$ & \\
\hline Group forming & $\checkmark$ & $\checkmark$ & & \\
\hline Exploration & $\checkmark$ & & & \\
\hline Endorsements & & $\checkmark$ & $\checkmark$ & $\checkmark$ \\
\hline
\end{tabular}

\section{A. Roles}

Users of REfine can fulfill three roles through their behavior: ideator (new needs are suggested for the system being studied), commenter (comments to existing needs are added), and assessor (voting on needs via agree/disagree). The proficiency in a role is represented by ideator points, commenter points and assessor points.

\section{B. Resources \& Points}

Points are directly earned by adding needs, commenting, and voting. Moreover, a participant obtains points indirectly when other participants vote or comment on or branch on her created needs. Coins (representing resources) have to be spent to perform all the actions but voting. This decision promotes constructiveness and lets users think carefully before sharing a need or comment. Table II shows the complete allocation of resources and points. We informally defined the amount of coins and points for the different actions. We ensured that need suggestion and branching required spending more coins, but also lead to rewards in terms of points (both active and passive). The most points are earned when receiving votes, which encourages valuable contributions.

Only the quantity of coins and points from active actions are communicated to the participants; the quantity of the passively earned or lost points are to discovered by the participants themselves, as an element of "surprise" of the game.

TABLE II

ALLOCATION OF RESOURCES AND POINTS ON REfine

\begin{tabular}{|l|l|l|}
\hline Action & \multicolumn{1}{|c|}{ Coins } & Points \\
\hline \multicolumn{3}{|c|}{ Active } \\
\hline Register & +10 & 0 \\
\hline Share a need & -3 & +5 ideator \\
\hline Comment & -1 & +1 commenter \\
\hline Vote & 0 & +1 assessor \\
\hline Branch & -3 & +5 ideator, +1 commenter \\
\hline \multicolumn{3}{|c|}{ Passive } \\
\hline Branched need & +1 & +3 ideator \\
\hline Voted comment & +1 & +1 commenter \\
\hline Agreed need & +1 & +1 ideator \\
\hline Disagreed need & 0 & -1 ideator \\
\hline
\end{tabular}

\section{Leaderboards}

Three types of leaderboards are used to increase motivation to participate, and to determine which stakeholders are to be invited to the focus groups for the selected requirements:

- Overall: a ranking based on accumulated points;

- Role-specific, each showing the ranking for ideator, commenter or assessor points;

- Need-specific, each focusing on one specific need. The ideator is marked by a badge.

\section{Group Formation}

Group formation is stimulated by the transparency of the stakeholders' background, and the separation of leaderboards per need. On the registration form, users select their role (e.g., developers, end-users), which is publicly shown on their profile. This transparency should clarify their perspective and increase the learning process among the different stakeholder groups. The separation of stakeholders per need defines groups based on the shared refinement of a specific need.

\section{E. Exploration}

Exploration is implemented minimally in REfine. Stakeholders can branch needs that were suggested by others and 
follow those traces. This allows exploring a need starting from its inception (the original need), and continuing by browsing the branches that are created. In future work, we plan to extend the exploration feature by allowing stakeholders to see how needs have evolved into product functionalities and releases.

\section{F. Endorsements}

While votes and comments are not typical game elements, they have the function of endorsements. These endorsements can confirm or oppose a need (votes), and provide useful information to improve a need (comments).

\section{THE CCRE METHOD}

REfine is part of the CCRE method for crowd-centric RE for software products. A high-level view on the method is in Fig. 3; all details can be found online [27].

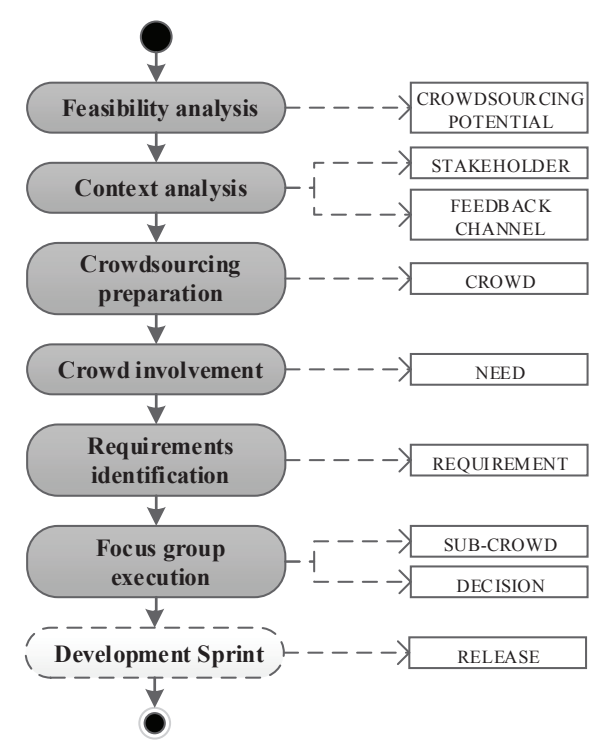

Fig. 3. The CCRE method. Although not shown, iterations exist both between and within the phases. Development sprint is not part of CCRE itself (hence, it is dashed). REfine supports the "Crowd involvement" phase

Feasibility analysis determines the applicability of CCRE, depending on the potential for crowdsourcing and gamification: is the SPO open to receive ideas from the users? These ideas may conflict with its vision and roadmap for the product.

Context analysis refines the scope and explicates the intended outcome. The stakeholders to involve in crowdsourcing are identified (users, developers, analysts, clients, regulatory bodies, etc.) and an interactive platform is selected as the primary feedback channel. A community manager is appointed to moderate the inputs and oversee the process.

Crowdsourcing preparation is about forming the crowd: stakeholders are recruited, through the provision of incentives to participate and the execution of a marketing campaign. To prevent misuse and chaos, guidelines on how to use REfine are developed and communicated to the crowd, and a specific scoping question is defined to keep the crowd focused on requirements for a well-defined aspect of the software product.
Crowd involvement is the phase where crowdsourcing happens, and that is supported by REfine. As per Sec. III, the crowd of stakeholders share, discuss, and refine their needs. To cope with stakeholders that bypass the primary channel, further feedback mining techniques should be adopted.

Requirements identification is conducted by the SPO: the collected needs are screened by product managers and requirements engineers, and a subset is mapped to possible requirements for the next product releases. In this phase, it is crucial not to overlook minority requirements, such as those that a small yet significant subset of the stakeholders required.

Focus group execution is intended to further develop the requirements by exploring alternative design options created by the SPO. In this phase, the most active stakeholders are invited to the focus group (as a reward for their help) including the ideator and most active contributors, are selected and invited to participate. During the focus groups, decisions are taken by reaching consensus among the participants.

\section{Evaluation: The Qubus Case Study}

We have conducted a case study where REfine was used to obtain requirements for the beta version release of the Qubus 7 software product. KPMG's Qubus ${ }^{4}$ is a Governance Risk and Compliance (GRC) web platform for compliance auditors to conduct their assessment activities at customers. Under the hood, Qubus embeds a workflow management system that enables users to customize the process behind compliance assessment. The focus of the RE exercise was on the usability for mobile devices, and current feedback included face-to-face interaction, phone calls and emails but were largely unused.

The stakeholders were invited to the platform in three rounds. First, we invited product managers and developers. Second, we contacted the clients and third, we invited the users related to the corresponding clients. In assessing the crowd, we found that the crowd lacked largeness (19 participants). We sent weekly updates to improve the activity of the members.

21 needs, 37 comments and 130 votes were shared over one month. Since the group of end-users remained largely inactive, we tuned the largeness of the crowd by inviting seven internal off-stage actors (potential end-users). Little moderation was needed, for all input was relevant to the asked question.

Three mainstream needs and one minority need were discussed by the SPO, the most important being "A loading screen should be shown when a page is loading". The 3 requirements with the highest relative priority were discussed in the focus group with the 5 top contributors according to the needspecific leaderboards of REfine. The identified solutions for two requirements were added to the product backlog.

\section{A. Effectiveness}

We report on two evaluations concerning the effectiveness of REfine in supporting elicitation and refinement. The former was with the REfine users of the Qubus 7 case study, and involved both our observation and a questionnaire concerning

\footnotetext{
${ }^{4}$ http://www.qubussoftware.com/
} 
TABLE III

NUMBER OF SHARED NEEDS, COMMENTS, VOTES AND EARNED POINTS PER STAKEHOLDER GROUP DURING THE CASE STUDY

\begin{tabular}{|l|r|l|r|l|r|r|r|r|r|}
\hline Stakeholder type & \multicolumn{1}{|c|}{$\mathbf{N}$} & \multicolumn{2}{|c|}{ Needs } & \multicolumn{2}{c|}{ Comments } & \multicolumn{2}{c|}{ Votes } & \multicolumn{2}{c|}{ Points } \\
\hline & & avg. & \multicolumn{1}{c|}{ tot. } & avg. & \multicolumn{1}{c|}{ tot. } & avg. & tot. & avg. & tot. \\
\hline Community manager & 1 & 2 & 2 & 8 & 8 & 21 & 21 & 41 & 41 \\
\hline Product management & 2 & 0 & 0 & 3 & 6 & 6 & 12 & 13.5 & 27 \\
\hline Development team & 4 & 0.8 & 3 & 3.8 & 15 & 14.8 & 59 & 26.8 & 107 \\
\hline Expert & 4 & 1 & 4 & 1.3 & 5 & 2.5 & 10 & 16 & 64 \\
\hline Client & 1 & 0 & 0 & 0 & 0 & 3 & 3 & 3 & 3 \\
\hline End-User & 1 & 1 & 1 & 0 & 0 & 3 & 3 & 11 & 11 \\
\hline Off-stage actor & 6 & 1.8 & 11 & 0.5 & 3 & 4.2 & 25 & 20 & 120 \\
\hline Total & 19 & 1.1 & 21 & 1.9 & 37 & 6.8 & 130 & 19.6 & 373 \\
\hline
\end{tabular}

their engagement, perceived ease of use and usefulness of REfine. The latter took place with internal (to KPMG) and external experts in product management and RE.

1) Evaluation with users of REfine: The points were unevenly distributed among the members of the crowd; $32 \%$ of the stakeholders earned more than $50 \%$ of the points, and $68 \%$ of the stakeholders earned $90 \%$ of the points. There was a large variety in the stakeholders' activity: the internal stakeholders that were not in the product management or development team suggested the most needs, which can be explained by their fresh perspective on the product. Most comments and votes were given by developers and product managers, probably due to their insight on the feasibility and their experience in product development. Table III displays the total and average activity of the various stakeholder groups in our case study.

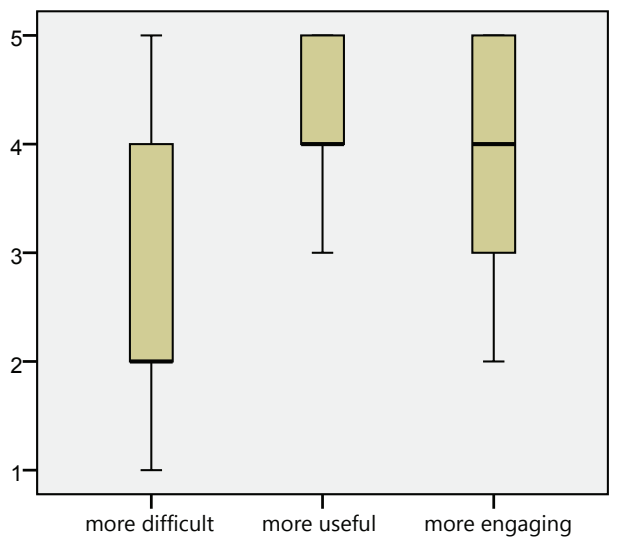

Fig. 4. REfine users: comparing the gamified, crowd-centric feedback experience with previous experiences

In the questionnaire, the 17 respondents found the process as difficult, more useful and more engaging compared with previous feedback experiences (Fig. 4). They felt motivated, thought their input would likely be taken into account, but they were not sure on the clarity of their priorities (Fig. 5). The most popular functions that the participants tried to achieve were "Read the needs of other participants" and "Provide suggestions for Qubus 7". Despite the fact that participants did not notice points and leaderboards prominently, they largely agreed that the game elements made the experience more pleasant. Voting and commenting were considered very useful, while the usefulness of branching was found neutral (Fig. 6).

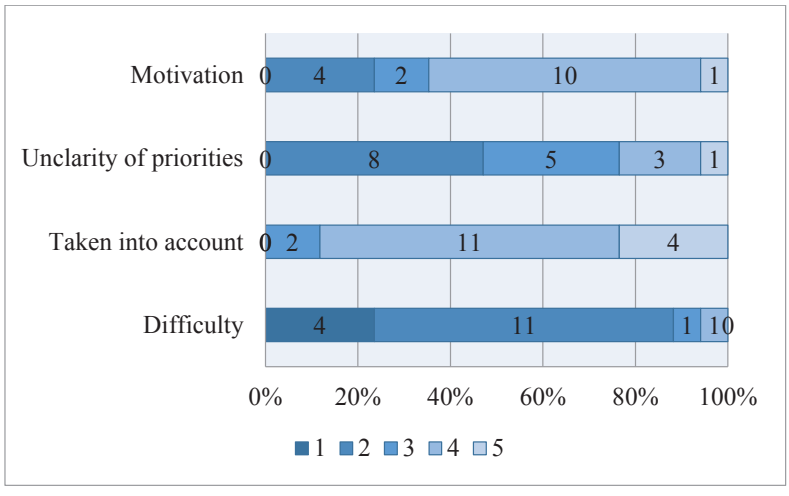

Fig. 5. REfine users: perceived experience during the feedback process

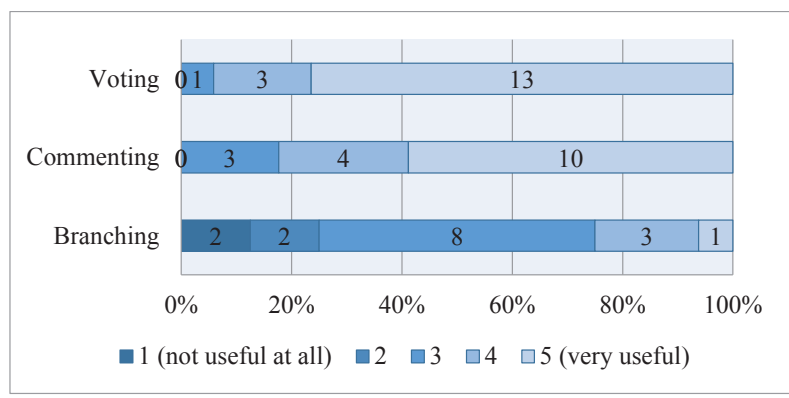

Fig. 6. REfine users: perceived usefulness of the functionality of REfine

2) Evaluation with experts: An interview with two members of the Qubus product management team revealed interesting insights. Advantages were approachability, structure and expected user adoption thanks to the method. Disadvantages that were identified included little incentive to return to the platform, no life cycle for the needs (i.e., needs are created, evolve, and disappear when implemented or excluded), and the risk that novice participants would suggest trivial needs. The number of needs was satisfying and the most important needs were considered detailed enough for use in a focus group.

The three consulted external experts-software product managers with experience in RE-responded on statements after a presentation of the method, prototype and resulting requirements. Table IV shows the ratings for the twelve statements they were confronted with. The experts found crowdsourcing useful for requirements elicitation, negotiation 
TABLE IV

RESULTS FROM THE EVALUATION WITH EXPERTS

\begin{tabular}{|l|c|c|c|}
\hline Statement & Exp. 1 & Exp. 2 & Exp. 3 \\
\hline Crowdsourcing useful for elicitation & 7 & 6 & 7 \\
\hline Crowdsourcing useful for negotiation & 6 & 5 & 6 \\
\hline Crowdsourcing useful for prioritization & 2 & 6 & 5 \\
\hline Crowdsourcing useful for specification & 6 & 5 & 7 \\
\hline Crowdsourcing useful for validation & 6 & 5 & 2 \\
\hline Gamification effective for engagement & 7 & 7 & 5 \\
\hline Gamification effective for innovation & 7 & 6 & 2 \\
\hline CCRE improves the process quality & 6 & 5 & 5 \\
\hline CCRE gives useful requirements & 6 & 6 & 7 \\
\hline CCRE gives higher quality requirements & 6 & 4 & 5 \\
\hline Detailed enough for focus group & 7 & 3 & 4 \\
\hline Detailed enough for Product Backlog & 7 & 2 & 4 \\
\hline
\end{tabular}

and specification and agreed that CCRE improves the quality of the RE process and gives a list of useful requirements. However, they think the quality of requirements would not be much better than the quality of the experts methods, and the requirements are maybe not detailed enough for a focus group or Product Backlog (contrary to what stated by the Qubus product managers). However, the experts commented that a meeting with the product management could improve this detail. Identified advantages included more feedback, openness and clear traceability and prioritization. Perceived disadvantages are the needed time to manage the method, the risk of a non-representative crowd, the difficulty to let developers and end-users have an effective dialog and the expectations that customers might have when needs are popular on the platform.

\section{DISCUSSION}

The quality of the RE process and of the requirements can be improved by opening participation to all stakeholders, including current and potential end-users, developers, and clients. The REfine tool helps by providing participation incentives via gamification. A case study showed the potential of the approach for improving RE in software production.

Our work contributes to the literature through a tool that aims to promote the long-term, sustainable collaboration among stakeholders. The focus of REfine is the clarification process of the identified needs, so to ease the SPO's job of turning them into system requirements.

Among the limitations, it is difficult to attract a large crowd that is a good sample of the active users, SPOs need to be transparent and open to discussion, and finding longterm incentives is hard. Our future work will tackle these limitations, also through the conduction of larger case studies.

\section{ACKNOWLEDGEMENTS}

This research is supported by a European FP7 Marie Curie grant (the SOCIAD Project).

\section{REFERENCES}

[1] D. E. Zand and R. E. Sorensen, "Theory of Change and the Effective Use of Management Science," Administrative Science Quarterly, vol. 20, no. 4, pp. 532-545, 1975 .

[2] S. Kujala, "User Involvement: A Review of the Benefits and Challenges," Behaviour \& Information Technology, 2003.
[3] S. Kujala, M. Kauppinen, L. Lehtola, and T. Kojo, "The Role of User Involvement in Requirements Quality and Project Success," in Proc. of RE, 2005, pp. $75-84$.

[4] L. Damodaran, "User Involvement in the Systems Design ProcessA Practical Guide for Users," Behaviour \& Information Technology, vol. 15 , no. 6, pp. 363-377, Jan. 1996.

[5] J. Kabbedijk, S. Brinkkemper, S. Jansen, and B. van der Veldt, "Customer Involvement in Requirements Management: Lessons from Mass Market Software Development," in Proc. of RE, 2009, pp. 281-286.

[6] The Standish Group, "CHAOS Summary 2009," Tech. Rep., 2009. [Online]. Available: http://emphasysbrokeroffice.com /files/2013/04/Standish-Group-CHAOS-Summary-2009.pdf

[7] J. Howe, "Crowdsourcing: A Definition," 2006. [Online]. Available: http://www.crowdsourcing.com/cs/2006/06/crowdsourcing_a.html

[8] S. Lim and A. Finkelstein, "StakeRare: Using Social Networks and Collaborative Filtering for Large-Scale Requirements Elicitation," IEEE Transactions on Software Engineering, vol. 38, no. 3, pp. 707-735, 2012.

[9] S. Lim, D. Damian, and A. Finkelstein, "StakeSource2.0: Using Social Networks of Stakeholders to Identify and Prioritise Requirements," in Proc. of ICSE, 2011, pp. 1022-1024.

[10] A. Adepetu, K. A. Ahmed, Y. A. Abd, A. A. Zaabi, and D. Svetinovic, "CrowdREquire: A Requirements Engineering Crowdsourcing Platform," in Proc. of the AAAI Spring Symposium: Wisdom of the Crowd, 2012.

[11] C. Eickhoff, C. G. Harris, A. P. de Vries, and P. Srinivassan, "Quality through Flow and Immersion: Gamifying Crowdsourced Relevance Assessments," in Proc. of ACM SIGIR, 2012, pp. 871-880.

[12] S. Deterding, D. Dixon, R. Khaled, and L. Nacke, "From Game Design Elements to Gamefulness: Defining Gamification," in Proc. of MindTrek: Envisioning Future Media Environments, 2011, pp. 9-15.

[13] J. Fernandes, D. Duarte, C. Ribeiro, C. Farinha, J. M. Pereira, and M. M. D. Silva, "iThink: A Game-Based Approach Towards Improving Collaboration and Participation in Requirement Elicitation," Procedia Computer Science, vol. 15, pp. 66-77, Jan. 2012.

[14] R. Snijders, F. Dalpiaz, M. Hosseini, A. Shahri, and R. Ali, "CrowdCentric Requirements Engineering," in Proc. of CGCloud, 2014.

[15] L. Cao and B. Ramesh, "Agile Requirements Engineering Practices: An Empirical Study," Software, IEEE, vol. 25, no. 1, pp. 60-67, 2008.

[16] D. C. Brabham, "Moving the Crowd at iStockphoto: The Composition of the Crowd and Motivations for Participation in a Crowdsourcing Application," First Monday, vol. 13, no. 6, 2008. [Online]. Available: http://pear.accc.uic.edu/ojs/index.php/fm/article/view/2159/1969

[17] J. Surowiecki, The Wisdom of Crowds. Random House LLC, 2005.

[18] R. Snow, B. O. Connor, D. Jurafsky, and A. Y. Ng, "Cheap and Fast-But is it Good?: Evaluating Non-Expert Annotations for Natural Language Tasks," in Proc. of EMNLP, 2008, pp. 254-263.

[19] A. Kittur, E. H. Chi, and B. Suh, "Crowdsourcing User Studies With Mechanical Turk," in Proc. of CHI, 2008, pp. 453-456.

[20] N. Kaufmann and D. Veit, "More than Fun and Money. Worker Motivation in Crowdsourcing-A Study on Mechanical Turk," in Proc. of AMCIS, 2011, pp. 1-11.

[21] D. Renzel, M. Behrendt, R. Klamma, and M. Jarke, "Requirements bazaar: Social requirements engineering for community-driven innovation," in Proc. of RE, Posters \& Demos. IEEE, 2013, pp. 326-327.

[22] M. Hosseini, K. Phalp, J. Taylor, and R. Ali, "Towards Crowdsourcing for Requirements Engineering," in Proc. of REFSQ Workshops, 2014.

[23] M. Hosseini, A. Shahri, K. Phalp, J. Taylor, R. Ali, and F. Dalpiaz, "Configuring Crowdsourcing for Requirements Elicitation," in Proc. of RCIS. IEEE, 2015.

[24] J. Hamari, J. Koivisto, and H. Sarsa, "Does Gamification Work? A Literature Review of Empirical Studies on Gamification," in Proc. of the HICSS, 2014, pp. 3025-3034.

[25] C. Ribeiro, C. Farinha, J. a. Pereira, and M. Mira da Silva, "Gamifying Requirement Elicitation: Practical Implications and Outcomes in Improving Stakeholders Collaboration," Entertainment Computing, vol. 5, no. 4, pp. 335-345, Jul. 2014.

[26] J. Hamari and J. Koivisto, "Social motivations to use gamification: an emperical study of gamifying exercise," in Proc. of ECIS, 2013.

[27] R. Snijders, O. Atilla, F. Dalpiaz, and S. Brinkkemper, "CrowdCentric Requirements Engineering: A Method based on Crowdsourcing and Gamification," Department of Information and Computing Sciences, Utrecht University, Tech. Rep. UU-CS-2015-004, 2015. [Online]. Available: http://www.cs.uu.nl/research/techreps/repo/CS2015/2015-004.pdf 